\title{
Corticosteroid in Septic Shock: Advantage or Disadvantage?
}

\author{
Sakura Muhammad Tola \\ Department of Pharmacology, Faculty of Medicine, YARSI University, Jakarta \\ Jalan Letjen. Suprapto, Cempaka Putih, Jakarta 10510 \\ Telepon (021) 4206674, 4206675, 4206676 \\ E-mail: sakura.muhammad@yarsi.ac.id
}

KEYWORDS sepsis, septic shock, hydrocortisone

ABSTRACT Sepsis has become a major health issue, with more patients dying in hospitals due to sepsis related complications. The pathogenesis of sepsis is complex and involves multiple aspects of the interaction between the infecting microorganisms and the host. Sepsis is fundamentally an inflammatory disease mediated by the activation of the innate immune system. In the case, sepsis is an unbalanced (proinflammatory and antiinflammatory), dysregulated response, the localized process goes out of control and becomes systemic contribute to diminished oxygen delivery. The process also leads to decreased hemodynamic stability, hyperglycemia and ultimately, multi organ dysfunction syndrome and death in septic shock. The use of corticosteroid hydrocortisone as adjunctive therapy is still debating due to the lack of supporting study and differences result among the studies.

\section{INTRODUCTION}

Sepsis was defined as the
systemic inflammatory response
syndrome (SIRS) caused by infection.
Shock septic is sepsis in which
circulatory, metabolic, and cellular
disorders are so severe that they
significantly increase mortality. The
prevalence of sepsis and septic shock is
steadily growing globally. Estimates of
the incidence of sepsis vary widely due to
differences in case ascertainment. Sepsis
was present in more than half of
hospitalizations ending in death or
terminal discharge to hospice in this
cohort of patients from 6 US hospitals,
and was the immediate cause of death in
most of these cases. However, most

underlying causes of death were associated with severe chronic comorbidities. One in 8 sepsis-associated deaths was potentially preventable through better hospital-based care, but only 1 in 25 sepsis-associated deaths was judged definitely or moderately preventable (Rhee et al. 2019)

The pathophysiology of sepsis is incompletely understood. There appears to be inappropriate regulation of normal physiological reactions to infection, both pro-inflammatory and anti-inflammatory, resulting in life-threatening organ dysfunction. The most severe form of sepsis is septic shock, a state of circulatory failure that occurs in a subset of patients with sepsis in whom circulatory, cellular and metabolic abnormalities are associated with an 
increased risk of death. The diagnosis of septic shock requires the presence of sepsis and hypotension requiring vasopressor therapy to maintain mean arterial pressure (MAP) $65 \mathrm{mmHg}$ or greater and a serum lactate of greater than $2 \mathrm{mmol} / \mathrm{L}$ despite adequate fluid resuscitation. In settings where lactate measurement is not available, other indices of tissue hypoperfusion, such as oliguria, altered mental status and delayed capillary refill, may be used instead. While hyperlactaemia is not a specific sign of sepsis and is not part of the definition of sepsis, it is a valuable marker of disease severity and remains an important component of many effective screening programmes and treatment algorithms (Thompson et al. 2018)

The treatment of sepsis should be based on the understanding of its pathogenesis. The pathogenesis of sepsis is not fully understood. Bacteria from external or local sources enter bloodstream causing bacteremia. Taking into account that phagocytosis in the bloodstream is impossible, blood humoral bactericidal factors and erythrocytes are probably the main antibacterial forces in the blood circulation. The humoral factors are more effective against bacteria from external source. Bacteria from a local source may be more resistant to the bactericidal action of plasma because blood humoral factors also present in the tissues and before entering the bloodstream from the tissues, bacteria should have overcome their action. Treating sepsis is a multidisciplinary task. Early recognition and commencing initial steps of resuscitation are inevitable to give the best possible chance for survival, which has to be started on the primary care level: outside the hospital, in the emergency department or on the wards. In the absence of adequate initial management, providing even the highest level of intensive care would be in vain.

\section{Pathophysiologic mechanisms in septic shock}

The pathogenesis of sepsis is complex and involves multiple aspects of the interaction between the infecting microorganisms and the host. The recognition of pathogens and the resulting cellular activation are fundamental for infection control. Paradoxically, the host inflammatory response is also the substrate for the pathophysiological changes in sepsis. Inflammation can cause dysfunction of the vascular endothelium, accompanied by cell death and loss of barrier integrity, giving rise to subcutaneous and bodycavity edema. In addition, mitochondrial damage caused by oxidative stress and other mechanisms impairs cellular oxygen use. Moreover, injured mitochondria release alarmins into the extracellular environment, including mitochondrial DNA and formyl peptides, which can activate neutrophils and cause further tissue injury (King et al., 2014)

Under normal conditions, bacterial entry into a sterile field activates resident macrophages to contain the initial infection. Oftentimes, the initial infectious insult is too great for resident macrophages alone to eradicate all bacteria. However, regardless of whether or not the primary infection is contained, macrophages, of the M1 phenotype, initiate the immune system response by releasing inflammatory mediators such as tumor necrosis factor (TNF), interleukin1b (IL-1b), IL-6 and CXCL-8. This signals endothelial cells to upregulate adhesion molecules and begin the recruitment of other inflammatory cells including the phagocytic cells (polymorphonuclear cells, monocytes and 
macrophages) of the innate immune response and lymphocytes of the adaptive immune response frequently become dysfunctional in sepsis to help control the infection. Neutrophils also have a role in cell recruitment signaling (King et al., 2014; Hotchkiss, et al., 2017).

Activation of these various receptors during infection is fundamental for the recognition of a wide range of microorganisms and result in complementary, synergistic, or antagonistic effects, thus modulating innate and adaptive immunity. The release of inflammatory mediators by innate immune cells upon pathogen recognition resulting in the activation of coagulation, vasodilation, endothelial leakage, rolling and extravasation of neutrophils and inflammatory mediators to the extravascular space, underscores the pathophysiology of organ dysfunctions and hypotension during sepsis. Of paramount importance, the inflammatory response triggers procoagulant factors, while natural anticoagulant factors, such as activated protein $\mathrm{C}$, anti-thrombin, and tissue factor inhibitors, are decreased in septic patients, resulting in a procoagulant state with multiple microthrombi and the obstruction of small vessels, which ultimately leads to intravascular disseminated coagulation and complement system, resulting in local ischemica, which impairs cellular respiration (Salomao et al., 2019). However, in the case of an unbalanced (proinflammatory and anti-inflammatory), dysregulated (maturation and proliferation) response, the localized process goes out of control and becomes systemic, in other words the disease of the whole body; hence, it gives way for impairing the function of distant vital organs. This makes the clinical manifestation of critical illness so similar regardless of the insult (Salomao et al., 201; Laszlo et al., 2015). The overall end result of these process are global tissue hypoxia in which the systemic oxygen delivery is insufficient to meet the oxygen demands of the body. This leads to decreased myocardial contractility, decreased systemic vascular resistance, hypotension, metabolic acidosis, hyperglycemia and ultimately, multi organ dysfunction syndrome and death.

\section{Diagnosis of Septic Shock}

The diagnosis of septic shock is multifactorial and includes: an initial assessment of the etiology and clinical signs and symptoms, of the hemodynamic parameters, of the cellular changes, and of the grade of tissue dysfunction. Some parameters of the definition of shock have not been clearly defined, e.g. adequate fluid resuscitation, no vasopressors, and threshold blood pressure. Rapid recognition of the underlying mechanism of shock is of paramount importance, as the delay in adequate treatment worsens patient's condition. Hemodynamic monitoring may help explain the patophysiological phenomena that are characteristic for septic shock is key principle in the management of the septic shock patient, resulting in improved survival outcomes (Angus et al., 2013). Deteriorating patients with a raised early warning score (such as a raised aggregate early warning score of 5 or above) should therefore be screened for infection. However, presently no gold standard to diagnose sepsis exists. It is fundamental to identify the signs of systemic inflammation, such as fever and leukocytosis, which may be the result of an infection process. Patients in septic shock present with impaired myocardial contractility in about $30 \%$ of 
cases. Investigations such as a complete blood count (CBC), urinalysis, blood cultures, chest $\mathrm{X}$-ray as well as more sophisticated imaging modalities may assist in finding the source of infection. Additional clinical and laboratory variables may be used to assess the severity of sepsis, organ dysfunction and organ hypoperfusion (Angus et al., 2013; Wadelek et al., 2017; Keeley et al., 2017).

\section{Treatment of Septic Shock}

The Surviving Sepsis Campaign, an international consortium of professional societies involved in critical care, treatment of infectious diseases, and emergency medicine, recently issued the clinical guidelines for the management of severe sepsis and septic shock. The important elements of the guidelines are organized into two "bundles" of care: an initial management bundle to be accomplished within 6 hours after the patient's presentation and a management bundle to be accomplished in the ICU. Implementation of the bundles is associated with an improved outcome. The principles of the initial management bundle are to provide cardiorespiratory resuscitation and mitigate the immediate threats of uncontrolled infection. The exact components required to optimize resuscitation, such as the choice and amount of fluids, appropriate type and intensity of hemodynamic monitoring, and role of adjunctive vasoactive agents, all remain the subject of ongoing debate and clinical trials. Nonetheless, some form of resuscitation is considered essential, and a standardized approach has been advocated to ensure prompt, effective management. The initial management of infection requires forming a probable diagnosis, obtaining cultures, and initiating appropriate and timely empirical antimicrobial therapy and source control (Angus et al., 2013; Dellinger et al., 2012).

\section{Pharmacokinetic Profile of Hydrocortisone}

Hydrocortisone is a member of glucocorticoids group. Gglucocorticoid activity is determined by a hydroxyl group at carbon-11 of the steroid molecule. Cortisone and prednisone are 11-keto compounds, lacking glucocorticoid activity. They are converted in the liver to cortisol and prednisolone respectively, the corresponding 11- $\beta$ hydroxyl compounds. All glucocorticoid preparations marketed for topical or local use (like intraarticular) are 11- $\beta$ hydroxyl compounds, obviating the need for biotransformation. The actions of all corticosteroids are mediated by interaction of hormone with corticosteroids receptor, which regulates gene transcription. Corticosteroids continue to act inside the cell even after their disappearance from the circulation, as the events initiated and the products of these events (such as specific proteins) may be present even after disappearance of corticosteroids from the circulation (Gupta et al., 2008).

Systemically used glucocorticoid are classified as short acting, intermediate acting and long acting based on their duration of ACTH suppression. They also differ in their relative glucocorticoid versus mineralcorticoid potency. However one must remember that even those corticosteroids which have low mineralcorticoid activity (eg. hydrocortisone) may have mineralcorticoid effects when used in high doses. The relative potency of corticosteroids differ due to their affinity for the receptor. However, observed potency is determined by both intrinsic biologic potency and duration of action. 
There is little correlation between circulating half life ( $\mathrm{t} 1 / 2)$ and its potency. Similarly little correlation exist between $\mathrm{t} 1 / 2$ and its duration of action (Gupta et al. 2008). The biologic rationale for their use includes immune modulation, effects upon cardiovascular tone, and the treatment of relative corticosteroid deficiency. The anti-inflammatory effects of corticosteroids are well established. Corticosteroids modulate the transcription of an array of mainly nuclear factor $\kappa \mathrm{B}$-regulated genes that contribute to inflammation. The synthesis of interleukin-1, interleukin-6, and tumor necrosis factor- $\alpha$ is inhibited, as is inducible cyclooxygenase 2 and inducible nitric-oxide synthase. GC decrease recruitment and function of inflammatory cells and vascular permeability at the site of inflammation. They also inhibit prostaglandin and leucotriene synthesis by inhibiting the release of arachidonic acid from the phospholipids. By these mechanisms, GC protect the organism from the damage caused by its own defense reactions and the products of these reactions during stress (Gupta et al., 2008).

Corticosteroids also enhance the vasoconstrictor response to vasopressor drugs, in particular exogenous catecholamines. Although the precise mechanism by which this occurs is not known, inhibition of cyclooxygenase 2 and inducible nitric-oxide synthase are likely to play a role. Corticosteroids also mediate catecholamine release from neural cells, and this may partly explain the effect of corticosteroids on the vasculature. Suppression of proinflammatory cytokines and improved circulatory dynamics provide biologic plausibility that corticosteroids may reduce mortality through improved tissue perfusion and metabolic function (Gupta et al., 2008; Cohen et al., 2019).

The physiological secretory rate of cortisol in the intact system is approximately $6 \mathrm{mg} / \mathrm{m} 2 /$ day. The usual maintainance glucocorticoid dose is adjusted above this estimated secretory rate as the bioavailability of cortisol is reduced by gastric acids and first pass metabolism in liver. Thus 8-10 $\mathrm{mg} / \mathrm{m} 2 /$ day of oral hydrocortisone (HC) is a reasonable initial starting dose, though patients with primary adrenal insufficiency may require slightly higher doses of $10-12 \mathrm{mg} / \mathrm{m} 2 /$ day. Later on, the dose may be individualised to avert signs and symptoms of adrenal insufficiency on the one hand while avoiding growth retardation and cushingoid features on the other. Continuous infusion of hydrocortisone could hasten the resolution of septic shock compared to bolus administration. Earlier initiation corresponds with a higher probability of shock reversal. Tapering strategy is unnecessary (Ibarra-Estrada et al., 2017).

\section{Study Related to the role of Corticosteroid Hydrocortisone in Septic Patients}

There is an established biological rationale for the administration of adjunctive corticosteroids in the management of patients with septic shock. Corticosteroids act through two mechanisms: immune modulation and cardiovascular modulation.

The Surviving Sepsis Campaign (SSC) in 2016 recommended that lowdose hydrocortisone should not be used as a routine adjunctive therapy in septic shock if adequate fluid resuscitation and vasopressor therapy restore hemodynamic stability. In patients who do not reach this goal, $200 \mathrm{mg}$ of hydrocortisone using continuous infusion 
per day should be given. The Surviving Sepsis Campaign recommended to administer hydrocortisone as continuous infusion to better prevent hyperglycemia. Rhodes et al., 2017 Nevertheless, the patterns in clinical practice remain widely heterogeneous because of differing interpretations of the definition of poor responsiveness of shock to fluid and vasopressor therapy, discrepancy between clinicians interpretation of guidelines, discrepancy in clinical practice, and unfamiliarity with existing evidence

The European multicenter (CORTICUS) study $(\mathrm{n}=499)$, which evaluated the role of $200 \mathrm{mg}$ of hydrocortisone/day versus placebo in patients with septic shock, did not demonstrate any beneficial effect on overall mortality $(34 \%$ vs. $31 \% ; P=$ $0.51)$ or in the subgroups of corticotropin "responders" or "nonresponders" and reported more episodes of superinfection in the patients who received steroids. 18 The CORTICUS trial had planned on a target enrollment of 800 patients to detect a $10 \%$ reduction in mortality in patients who were nonresponders. The study was stopped prematurely when lower than expected recruitment resulted in termination of funding and expiry of the study drug supply. As a result, the trial was significantly underpowered to detect a clinically important treatment effect (Sprung et al., 2008). Lian et al conducted study meta-analysis to reevaluate the benefits and risks of corticosteroid treatment in adult patients with septic shock. Twenty-one RCTs were identified and analyzed. Patients treated with corticosteroid had a $7 \%$ reduction in relative risk in 28-day allcause mortality compared to controls (RR $0.93,95 \%$ CI 0.88 to 0.99 ). However, there were no significant differences for the intensive care unit (ICU) mortality (RR $0.97,95 \%$ CI 0.86 to 1.09 ) or inhospital mortality (RR 1.01, 95\% CI 0.92 to 1.11). Corticosteroids shortened the length of ICU stay by 1.04 days (RR $1.04,95 \% \mathrm{CI}-1.72$ to -0.36 ) and the length of hospital stay by 2.49 days (RR $2.49,95 \%$ CI -4.96 to -0.02 ). Corticosteroids increased the risk of hyperglycemia (RR 1.11, 95\% CI 1.06 to 1.16) but not gastroduodenal bleeding (RR 1.06, 95\% CI 0.82 to 1.37 ) or superinfection (RR $1.04,95 \%$ CI 0.94 to 1.15). However, some date on secondary outcomes were unavailable because they were not measured or not reported in the included studies which may cause a lack of power or selective outcome reporting. The information size was calculated at 10044 patients. Trial sequential analysis showed that the meta-analysis was conclusive and the risk of type 2 error was minimal. The study concluded that corticosteroids are likely to be effective in reducing 28-day mortality and attenuating septic shock without increasing the rate of life-threatening complications. TSA showed that the risk of type II error in this meta-analysis was minimal and the result was conclusive (Lian et al. 2019).

The adrenal trial randomized 3,800 patients with septic shock in 69 intensive care units from five countries to either $200 \mathrm{mg}$ of hydrocortisone administered by infusion or matched placebo. There was no statistically significant difference in the primary outcome of 90-day mortality between the two groups $(27.9 \%$ vs. $28.8 \%$; OR, 0.95; CI, 0.82 to $1.1 ; P=0.5)$. However, some of the secondary outcomes were improved in the hydrocortisone group; patients assigned to the hydrocortisone group had earlier shock reversal, faster liberation from mechanical ventilation, reduced 
frequency of blood transfusion, and earlier discharge from intensive care. There were no significant differences between the treatment groups with respect to 28-day mortality, the rate of recurrence of shock, recurrence of mechanical ventilation, duration and rate of use of renal replacement therapy, time to hospital discharge, the rate of development of new-onset bacteremia or fungemia, and 6-month mortality (Venkatesh et al., 2018).

The second double-blind RCT (APROCCHSS) by Djillali Annane investigated the combination of intravenous bolus hydrocortisone $(50 \mathrm{mg}$ four times a day) and oral fludrocortisone (50 $\mu \mathrm{g}$ per day) in 1,241 patients. For inclusion in the study the use of vasopressors (norepinephrine, epinephrine) was required at a minimum dose of $\searrow 0.25 \mu \mathrm{g}$ per kilogram of body weight per minute or $\geq 1 \mathrm{mg}$ per hour for at least 6 hours to maintain a systolic blood pressure of at least $90 \mathrm{mmHg}$ or a mean blood pressure of at least $65 \mathrm{mmHg}$. The patients was divided into the groups receiving Hydrocortisone $50 \mathrm{mg}$ IV every 6 hours and fludrocortisone $50 \mathrm{mcg}$ NG daily for 7 days without taper and the group receiving placebo. In this study, a significant reduction in death of any cause at day 90 was reported with the use of steroids $(43.0 \% v s .49 .1 \%, \mathrm{P}=0.03)$. In addition, mortality was reduced at ICU discharge $(35.4 \%$ vs. $41.0 \%, \mathrm{P}=0.04)$, hospital discharge $(39.0 \%$ vs. $45.3 \%$, $\mathrm{P}=0.02$ ), and day 180 (46.6\% vs. 52.5\%, $\mathrm{P}=0.04)$. Secondary endpoints such as vasopressor-free days and organ-failurefree days to day 28 were significantly higher in patients treated with the two steroids, as was the time to weaning from mechanical ventilation to day 90 (Annane et al., 2018).

Overall corticosteroids are typically inexpensive and widely available. The impact of corticosteroids on the overall costs to patients and to health systems is uncertain and would be driven mostly by ICU and hospital lengths of stay or prolonged periods of rehabilitation. Beside that corticosteroid treatment is associated with numerous adverse effects, including metabolic derangements, gastrointestinal bleeding, neuromuscular weakness, and immunosuppression. A tapering strategy for cessation of corticosteroids has previously been advocated because of the observation of an increase in inflammatory mediators upon abrupt cessation of treatment.

\section{CONCLUSION}

Septic shock is an emergency state that requires a rapid diagnostic process that helps to discover signs and symptoms and the etiology of the shock. The role of adjunctive corticosteroid in septic shock patient remains controversial. Although some studies showed the administration of hydrocortisone could improve the survival rate of the patients. Clinical questions remain over the optimum duration of treatment and the role of corticosteroids in relapsing shock. The use of hydrocortisone must be justify by the clinician based on the patient condition. More studies are needed to clarify and intensify the role of corticosteroid and the impact of corticosteroid therapy on healthcare cost in septic patient in the intensive care units. 


\section{REFERENCES}

Angus, DC, van der Poll T. 2013. Severe Sepsis and Septic Shock. N Engl J Med 369:840-51.

Annane, D., Renault, A., Brun-Buisson, C., Megarbane, B., Quenot, J.P., Siami, S. 2018. e Hydrocortisone plus Fludrocortisone for Adults with Septic Shock. N Engl J Med. 378(9):809-8.

Cohen, J., Venkatesh, B. 2019. Adjunctive Corticosteroid Treatment in Septic Shock. Anesthesiology. 131:410-9.

Dellinge,r RP, Levy MM, Rhodes A, Annane D, Gerlach H, Opal SM, et al. 2013. Surviving Sepsis Campaign: International Guidelines for Management of Severe Sepsis and Septic Shock: 2012. Crit Care Med. 41:580-637.

Gupta, P, Bhatia, V. 2008. Corticosteroid Physiology and Principles of Therapy. Indian J Pediatr. 75 (10) : 1039-1044.

Hotchkiss, R.S., Moldawer, L.L., Opal, S.M., Reinhart, K., Turnbull, I.R., Vincent. J.L. 2017. Sepsis and septic shock. Nat Rev Dis Primers. Author manuscript; available in PMC 2017 August 01.

Ibarra-Estrada, M.A., Chávez-Peña, Q., Reynoso-Estrella, C.I., RiosZermeño, J., Aguilera-González, P.E., García-Soto, M.A., et al. 2017. Timing, method and discontinuation of hydrocortisone administration for septic shock patients. World J Crit Care Med 6:1:65-73

Keeley, A., Hine, P., Nsutebu, E. 2017. The recognition and management of sepsis and septic shock: a guide for non-intensivists. Postgrad Med $\mathrm{J} ; 93: 626-634$.
King, E.G., Bauza, G.J., Mella, J.R., Remick, D.G. Pathophysiologic mechanisms in septic shock. Laboratory Investigation 2014;94:4-12.

Lian, XJ, Huang, DZ, Cao, YS, Wei, YX, Lian ZZ, Qin TH, et al. 2019. Reevaluating the Role of Corticosteroids in Septic Shock: An Updated Meta-Analysis of Randomized Controlled Trials. BioMed Research International. Article ID 3175047, 14 pages https://doi.org/10.1155/ 2019/3175047.

Rhee, C, Jones, TM, Hamad, Y, Pande ,A, Varon, J, O Brien, C, et al. 2019. Prevalence, Underlying Causes, and Preventability of SepsisAssociated Mortality in US Acute Care Hospitals. JAMA Netw Open. 2(2):e187571.

Rhodes, A., Evans, L.E., Alhazzani, W., et al. 2017. Surviving Sepsis Campaign: International Guidelines for Management of Sepsis and Septic Shock: 2016. Crit Care Med 2017;45:486-552.

10.1097/CCM.0000000000002255.

Salomao, R., Ferreira, B.L., Salomao, M.C., Santos, S.S., Azevedo, L.C.P., Brunialti, M.K.C. 2019. Sepsis: evolving concepts and challenges. Brazilian Journal of Medical and Biological Research 52(4): e8595.

Sprung, CL, Annane, D, Keh, D, Moreno, R, Singe,r M, Freivogel, K, et al. 2008. Hydrocortisone Therapy for Patients with Septic Shock. N Engl J Med. 358:111-24.

Thompson, K, Venkatesh, B, Finfer, S. 2019. Sepsis and septic shock: current approaches to management. Internal Medicine Journal 49:160170. 
Venkatesh, B, Finfer, S, Myburgh, J, Cohen, J, Billot, L. 2018. Longterm outcomes of the ADRENAL Trial. N Engl J Med. 378:1744-5.
Wadelek, J. 2017. Diagnosis and Treatment of Patients in Septic Shock. New Med. 21(1): 31-36. 